Dementia and Risk of Adverse Warfarin-Related Events in Nursing Homes

\title{
Comparative Rates of Adverse Events With Parenteral Formulations of Iron
}

\author{
Joel Shuster, PharmD, BCPP*
}

\begin{abstract}
The purpose of this feature is to heighten awareness of specific adverse drug reactions (ADRs), discuss methods of prevention, and promote reporting of ADRs to the US Food and Drug Administration's (FDA's) MEDWATCH program (800-FDA-1088). If you have reported an interesting, preventable ADR to MedWatch, please consider sharing the account with our readers. Write to Dr. Shuster at ISMP, 200 Lakeside Drive, Suite 200, Horsham, PA 19044 (phone: 215-947-7797; fax: 215-914-1492; e-mail: joel.shuster@temple.edu). Your report will be published anonymously unless otherwise requested. This feature is provided by the Institute for Safe Medication Practices (ISMP) in cooperation with the FDA's MedWatch program and Temple University School of Pharmacy. ISMP is an FDA MEDWATCH partner.
\end{abstract}

*Clinical Professor of Pharmacy Practice, Temple University School of Pharmacy, Philadelphia, Pennsylvania; Clinical Consultant, Episcopal Hospital, Philadelphia, Pennsylvania; Clinical Advisor, Institute for Safe Medication Practices, Horsham, Pennsylvania. 


\section{AGRANULOCYTOSIS INDUCED BY PROTON PUMP INHIBITORS}

A 20-year-old man with a history of cystic fibrosis was treated with tobramycin, meropenem, and teicoplanin for a respiratory infection over a 2 -week period. He had been treated with these agents "regularly" in the past without any hematologic toxicity. He had also been taking omeprazole for almost 4 years. Less than 2 weeks after completing his antibiotic therapy, he presented to the hospital in septic shock with agranulocytosis. His white blood cell (WBC) count was measured at $0.7 \mathrm{x}$ $10^{9}$ cells $/ \mathrm{L}$ (reference range, $4.5-11 \times 10^{9} / \mathrm{L}$ ) while the neutrophils were less than $0.1 \times 10^{9} / \mathrm{L}$ (reference range, $\left.1.8-6.5 \times 10^{9} / \mathrm{L}\right)$. The omeprazole was discontinued, ranitidine was added, and similar antibiotics were restarted. Within 48 hours, the patient's WBC count and clinical picture improved. Ten days later, the WBC count was $13.4 \times 10^{9} / \mathrm{L}$ and the patient was discharged soon after.

Esomeprazole (Nexium) had been given to the patient a few days before discharge because his reflux symptoms had returned. Sixteen days after the esomeprazole had been added at $40 \mathrm{mg}$ daily, the patient returned to the hospital with fever and a recurrence of agranulocytosis with the total WBC count at $1 \times 10^{9} / \mathrm{L}$ and the neutrophils less than $0.1 \times 10^{9} / \mathrm{L}$. The esomeprazole was immediately halted and antibiotics were initiated. Three days later, there was a significant improvement in both the total WBC and neutrophil counts. The patient was not given proton pump inhibitors (PPIs) again.

The authors investigated the patient's cytochrome P450 enzyme activity and discovered that he had a "homozygotous mutation of CYP2C19*17." This enzyme has been identified as being responsible for the metabolism of PPIs and some antidepressants. The authors surmised "that the enhanced enzyme activity may have induced an increase of toxic PPI metabolites leading to agranulocytosis."

Dury S, Nardi J, Gozalo C, Lebargy F, Deslee G. Agranulocytosis induced by proton pump inhibitors. J Clin Gastroenterol. 2012;46(10):859.

\section{TWO CASES OF FATAL ANTIMALARIAL- INDUCED CARDIOMYOPATHY}

The authors of this report tell us that antimalarial drugs used in patients with rheumatologic diseases may cause myopathies in $10 \%$ of patients or more. Both cardiac and skeletal muscles may be involved. Following is a review of 1 of 2 cases presented where patients died from cardiomyopathy related to the use of chloroquine or hydroxychloroquine.
A 38-year-old woman had been taking chloroquine $500 \mathrm{mg}$ daily for 7 years. Her diagnosis was discoid lupus erythematosus. Over the prior 7 months, she had developed "progressive muscle weakness" that was especially noticeable in her extremities. She had undergone a recent liver biopsy for mildly elevated liver transaminases with negative biopsy findings. She presented with dizziness and syncopal episodes and was found to have congestive heart failure with a "highgrade atrioventricular (AV) block." Echocardiography demonstrated a dilated cardiomyopathy, and a biopsy of her right ventricle revealed "patchy vacuolar changes" with interstitial infiltration of lymphocytes. There was no evidence of glycogen or myeloid infiltration. Because of continuing progression of muscle weakness, the woman underwent electromyography which showed myopathic features of both the upper and lower extremities. The serum creatine kinase level was measured at 652 units/L (reference range, 30-300 units/L). Because of worsening AV block, the patient required the placement of a pacemaker/implantable cardioverter defibrillator. She showed temporary improvement, but 2 months later she declined rapidly and was readmitted in an hypoxic state with cardiogenic shock. The chloroquine was finally stopped, but the patient died 5 days later. The autopsy showed widespread myopathic changes in the heart muscle, deltoids, and quadriceps. The changes were consistent with changes seen in myopathies caused by chloroquine and hydroxychloroquine.

The authors offer a concise review of the potential of myotoxicity related to these antimalarial agents and tell the reader that there are reports of such cardiomyopathy being reversible within 2 to 9 months after discontinuation of the offending agent. It is recommended that patients be screened for possible muscle enzyme disturbances when treated long term with these 4-aminoquinolone derivatives.

Azimian M, Gultekin SH, Hata JL, Atkinson JB, Ely KA, Fuchs HA, et al. Fatal antimalarial-induced cardiomyopathy - report of 2 cases. J Clin Rheumatol. 2012;18(7):363-366.

\section{OXALIPLATIN-INDUCED THROMBOTIC THROMBOCYTOPENIC PURPURA}

A 68-year-old woman developed rectal adenocarcinoma. At the initial diagnosis, she was found to have metastases to her liver. She was treated with the FOLFOX regimen of infusional fluorouracil, leucovorin, and oxaliplatin for 4 cycles before she underwent surgery, leaving her with an ileostomy. She was treated again with FOLFOX and bevacizumab before having a resection of her liver. New lung metastases necessitated further therapies until she was somewhat stable. After another 
year and a half, she demonstrated lung and liver nodules and was treated with cryotherapy. Because of continued progression of her disease, she was again treated with FOLFOX, almost 2 years after receiving that combination of antineoplastic agents at the start of her ordeal.

The patient tolerated her first re-retreatment without a problem, but on the first day of her second cycle, she "developed sudden-onset chest pain" after only onehalf of the infusion of oxaliplatin. The infusion was stopped, and treatment in the emergency department (ED) with aspirin and nitroglycerin attenuated her chest pain. A cardiac workup found no evidence of a myocardial infarction. The patient was admitted and her condition worsened overnight when she started to bleed from her ileostomy. Laboratory evaluation showed anemia with severe thrombocytopenia. The platelet count had fallen in 2 days from $119 \times 10^{9}$ cells/L to just $31 \times 10^{9} / \mathrm{L}$ (reference range, 150-450 x 10\%/L). The serum creatinine, bilirubin, lactate dehydrogenase, and serum transaminases were all markedly higher than the pretreatment values. There was obvious laboratory evidence of severe hemolysis. All of these findings, along with the patient's fever and confusion, led to the diagnosis of thrombotic thrombocytopenic purpura (TTP). The patient was treated with high doses of prednisone and plasma exchanges. Sixteen days later, her hemoglobin had stabilized and her platelet count was much improved without further evidence of hemolysis.

The authors tell us that prior reports have shown cases of "infrequent late-onset allergic reaction in some patients" treated with oxaliplatin and there have been reports of thrombocytopenia or hemolysis with oxaliplatin previously, but that this may be the first report of TTP associated with the drug, probably due to an immune-mediated mechanism.

Niu J, Mims MP. Oxaliplatin-induced thrombotic thrombocytopenic purpura: case report and literature review. J Clin Oncol. 2012;30(31):e312-e314.

\section{UNIQUE SKIN ERUPTION CAUSED BY TELAPREVIR}

This report opens with the reminder that telaprevir (Incivek) is known to cause nonspecific skin eruptions at a rate of approximately $40 \%$ to $60 \%$ in various treatment groups. This agent is a protease inhibitor of the hepatitis $\mathrm{C}$ virus (HCV).

A 55-year-old man began treatment for HCV with telaprevir, pegylated interferon alfa- $2 \mathrm{a}$, and ribavirin. Six weeks later, he began to develop "generalized, salmonred erythema with coarse desquamation and islands of sparing on the trunk, extremities, head, neck, and soles." After 12 more weeks of progressive discomfort, the patient presented to his clinic where physical exami- nation did not show any lymphadenopathy or involvement of the patient's mucosa or palms. The patient complained of malaise, skin dysesthesias, pruritus, and chills. The eosinophil count was markedly elevated, and the patient was thrombocytopenic. The serum creatinine had increased from a baseline of $1.14 \mathrm{mg} / \mathrm{dL}$ (reference range, $0.5-1.4 \mathrm{mg} / \mathrm{dL}$ ) to $1.8 \mathrm{mg} / \mathrm{dL}$. Skin biopsy showed a marked "resemblance to pityriasis rubra pilaris (PRP) as opposed to a classic erythematous morbilliform druginduced skin eruption" that is the usual presentation of the dermatologic adverse effects seen with telaprevir. Because the patient had previously been treated with the ribavirin and interferon, the addition of the telaprevir was the determining factor in its being blamed for this rare adverse effect of a PRP-like problem. The authors state that only sorafenib has been previously implicated as a causative agent for drug-induced PRP-like cutaneous eruptions.

Stalling SS, Vu JR, English JC. Telaprevir-induced pityriasis rubra pilaris-like eruption. Arch Dermatol. 2012;148(10):1215-1217.

\section{ACUTE-ONSET OPIOID-INDUCED HYPERALGESIA IN A CHILD}

An 8-year-old girl developed "dull aching pain in her knees and ankles associated with morning stiffness and synovitis." After experiencing such symptoms for 6 months, she was seen at a rheumatology clinic and diagnosed with polyarticular juvenile idiopathic arthritis. She was started on methotrexate and naproxen. Because of continued joint pain and swelling, etanercept was added 4 months later. She was admitted to a community hospital 1 month later because of sore throat, fever, headache, and bilateral hip pain. She was given antibiotics for presumed sinusitis, but the "dull aching pain" in her hips continued even though she continued taking naproxen. She did not complain of knee or ankle pain. She described the pain level as 5 out of 10 .

The patient was discharged, but soon after was taken to another local hospital because of increasing hip pain. She was given morphine $2 \mathrm{mg}$ intravenously (IV) along with ketorolac and soon after she "developed shooting pain in the hips, arms, and shoulders." The child described the pain as, "It feels like my bones are breaking." She stated that this new pain was unlike the joint pain that she had been dealing with for months. She actually refused to sit up or bear weight because of the severity of the pain. She was then placed on patientcontrolled analgesia (PCA) with hydromorphone and was also given lorazepam for agitation. Neither of these therapies changed the intensity of her pain. After the second day of the unrelenting pain, she was transferred to a tertiary care center for further evaluation. The pain scores remained in the range of 8 to 10 on the scale of 10 . 
The patient was continued on the same analgesic regimen at the new hospital upon admission, but because of worsening pain scores the pain medication was switched to PCA with fentanyl. The pain scores did not change and the patient "was noted to be sensitive to even slight touch over her upper and lower extremities and also sensitive to sound and light."

She was described as being more agitated and distressed and was tachycardic. She still described her pain as though her bones were breaking. At this point, she underwent a workup looking for possible neoplasms that included computed tomographic scans, ultrasound studies, and magnetic resonance imaging. She had a bone marrow biopsy looking for lymphoma or leukemia. All tests were negative.

The pediatric intensive care unit was consulted and after discussion with the patient's family, the decision was made to stop the narcotic infusion and continue with the ketorolac only. Two days later, "there was a dramatic improvement" in the patient's pain. Soon after, she was discharged on celecoxib with mild complaints of the type of arthritic pain that she had experienced previously.

The authors describe this case as one of opioidinduced hyperalgesia $(\mathrm{OIH})$. This patient suffered much more severe pain right after she was given morphine in her local hospital, and it did not remit until the opioids were discontinued days later. A brief discussion of $\mathrm{OIH}$ is presented along with a consenting editorial in the same issue of the Journal of Clinical Rheumatology.

Vijayan V, Moran R, Elder ME, Sukumaran S. Acute-onset opioid-induced hyperalgesia in a child with juvenile idiopathic arthritis. J Clin Rheumatol. 2012;18(7):349-351.

Lee MO. Opioid-induced hyperalgesia [editorial]. J Clin Rheumatol. 2012;18(7):325-326.

\section{RISK OF SHARING A PILL CUTTER}

A 45-year-old woman went to her internist after suffering various symptoms for almost a month. She had complaints of nausea, weakness, and decreased appetite causing a $4-\mathrm{kg}$ weight loss. The problems started about a week after her dog had died. She was given a series of tests, and it was determined that she had adrenal insufficiency which was secondary to adrenocorticotropin hormone (ACTH) suppression. She denied the use of any glucocorticoids orally, topically, or parenterally.

Further investigation found that the patient had been using a pill cutter to cut her daily antidepressant medication and her dog's prednisone tablets. The pet had been treated for Addison's disease for 6 years. The woman had been cutting $5 \mathrm{mg}$ prednisone tablets into quarters twice daily for all of those years and was getting enough of a dose of prednisone herself to suppress her own production of cortisol. When the dog had died, she stopped "getting" her daily dose of prednisone pieces and developed the secondary adrenal insufficiency. She was placed on hydrocortisone therapy which was tapered off over 7 weeks or so. She had no further sequelae.

Thapa S, Salvatori R. The risk of pill cutter sharing. Am J Med. 2012;125(11):e7.

\section{DEMENTIA AND RISK OF ADVERSE WARFARIN-RELATED EVENTS IN NURSING HOMES}

A prospective cohort study of patients in 26 nursing homes showed that patients with dementia were much more likely to suffer adverse warfarin events than patients without a diagnosis of dementia. There was a consistent increase in nonpreventable and preventable or potential adverse events with warfarin therapy in the dementia population. The study also demonstrated that the patients with dementia had the same amount of monitoring tests and days in therapeutic INR ranges as nondementia patients. Results showed that greater nursing staff time equated to a lower risk of preventable adverse events. The authors surmise that increased infections and the probable use of more antibiotics in patients with advanced dementia may be a factor in the increased adverse events in this group.

Tjia J, Field TS, Mazor KM, et al. Dementia and risk of adverse warfarin-related events in the nursing home setting. Am J Geriatr Pharmacother. 2012;10(5):323-330.

\section{COMPARATIVE RATES OF ADVERSE EVENTS WITH PARENTERAL FORMULATIONS OF IRON}

The authors of this retrospective review looked at the use of 4 different formulations of IV iron products over a 2-year period at 6 hospitals in the Boston area. (There are 5 iron preparations available for IV use in the United States, but only 4 were used at this hospital system.) A total of 619 unique patients were studied. The study showed only 32 adverse events, and they were all described as mild or moderate. The results showed a "significantly higher odds ratio of adverse events" associated with the iron sucrose (Venofer) formulation compared to the other agents. Only $15.5 \%$ of the patients were treated with this product. The authors recommend increased use of the low-molecular-weight iron dextran $(\mathrm{INFeD})$ product, which can be given as a total-dose infusion, although they point out that the adverse event rates are acceptable across the board for this class of drugs.

Okam MM, Mandell E, Hevelone N, Wentz R, Ross A, Abel GA. Comparative rates of adverse events with different formulations of intravenous iron. Am J Hematol. 2012;87(11):e123-e124. 